\title{
Correction to: A post-processing scheme for the performance improvement of vehicle detection in wide-area aerial imagery
}

\author{
Xin Gao ${ }^{1}$. Sundaresh Ram² . Jeffrey J. Rodríguez ${ }^{1}$
}

Published online: 5 March 2020

(c) Springer-Verlag London Ltd., part of Springer Nature 2020

\section{Correction to:}

\section{Signal, Image and Video Processing https://doi.org/10.1007/s11760-019-01592-4}

1. In the original publication, the co-authors' names were omitted. The correct list of authors is the following:

Xin $\mathrm{Gao}^{1}$, Sundaresh Ram ${ }^{2}$, and Jeffrey J. Rodríguez ${ }^{1}$

${ }^{1}$ Department of Electrical and Computer Engineering, The University of Arizona, Tucson, AZ, USA

${ }^{2}$ Department of Radiology, Department of Biomedical Engineering, University of Michigan, Ann Arbor, MI, USA

2. Change "binary masking" to "filtered dilation" in Section 3 at item (1), in Table 3, in Figs. 6 and 7, and at the two occurrences in the text of Section 5.

3. In Section 3, in the Step 1 paragraph, revise the third sentence as follows:

For example, we used $t_{1}=t_{a v g} / 20$ and $t_{2}=\max$ $\left(6 * t_{\text {avg }}, 2 * t_{\text {large }}\right)$, corresponding to the traffic congestion case of six vehicles clustered together or two largest vehicles being merged.

4. In Section 4, reword Step 5 as follows:

Label the largest non-zero element in each column as a TP.

5. In Table 1, change "Time/s" to "Time (s)".

6. In the Table 3 caption, change " $10 \times 10$ " to " $100 "$.

The original article can be found online at https://doi.org/10.1007/ s11760-019-01592-4.

Xin Gao

xgao1985@email.arizona.edu

1 Department of Electrical and Computer Engineering, The University of Arizona, Tucson, AZ, USA

2 Department of Radiology, Department of Biomedical Engineering, University of Michigan, Ann Arbor, MI, USA
7. In the Fig. 4 caption, change "left" to "top" and "right" to "bottom".

8. Delete Fig. 5 and Table 2, along with the paragraph in Section 5 which refers to them, and delete the last clause of the third sentence in Section 6.

9. Add the following references:

46. Ram, S., Rodríguez, J.J.: Vehicle detection in aerial images using multiscale structure enhancement and symmetry. In: 23rd IEEE International Conference on Image Processing (ICIP), pp. 3817-3821 (2016)

47. Ram, S.: Sparse Representations and Nonlinear Image Processing for Inverse Imaging Solutions. Ph.D. Dissertation, Department of Electrical and Computer Engineering, The University of Arizona, Tucson, AZ, USA (2017)

Publisher's Note Springer Nature remains neutral with regard to jurisdictional claims in published maps and institutional affiliations. 